\title{
Explorer les liens entre agriculture, migration et sécurité alimentaire: une enquête auprès de ménages agricoles diversifiés et multilocalisés du nord-ouest du Nicaragua
}

\author{
Sandrine Fréguin-Gresh ${ }^{1,7, *}$, Lissania Padilla ${ }^{2}$, Karen Pavon $^{2}$, Douglas Elizondo ${ }^{3}$, Geneviève Cortès ${ }^{4,7}$, \\ Valentina Banoviez Urrutia ${ }^{4}$, Alissia Lourme Ruiz ${ }^{5}$, Sandrine Dury ${ }^{6,7}$ et Emmanuelle Bouquet ${ }^{6,7}$ \\ 1 CIRAD, UMR ART-DEV, 34398 Montpellier, France \\ 2 Instituto de Investigación y Desarrollo NITLAPAN, Managua, Nicaragua \\ ${ }^{3}$ Sustainable Science Institute, Managua, Nicaragua \\ ${ }^{4}$ UMR ART-DEV, Université Paul Valéry, Montpellier, France \\ ${ }^{5}$ UMR Nutripass, IRD, Université de Montpellier, SupAgro, Montpellier, France \\ ${ }^{6}$ CIRAD, UMR MOISA, 34398 Montpellier, France \\ ${ }^{7}$ Univ Montpellier, Montpellier, France
}

\begin{abstract}
Résumé - Cet article présente une enquête menée dans le nord-ouest du Nicaragua, qui vise à comprendre comment les flux d'argent et d'aliments générés par les activités diversifiées et multilocalisées des familles agricoles interviennent dans l'accès aux aliments et dans la perception de la sécurité alimentaire. Dans la région d'étude, l'agriculture familiale est caractérisée par des conditions agro-écologiques difficiles et repose sur la production vivrière et l'élevage bovin. En réponse au manque de revenus, les ménages ont depuis longtemps diversifié leurs activités hors exploitation, localement ou en migration. Cette diversification et cette multilocalisation leur permettent d'obtenir des revenus et des aliments, indispensables à la sécurité alimentaire dans les régions d'origine. Ces flux d'argent et d'aliments se fondent sur des rapports sociaux entre les membres des ménages et au-delà (famille élargie, voisinage, acteurs institutionnels). Pour capter ces flux, le questionnaire d'enquête, composé de trois sections, s'organise autour d'une clef d'identification unique : l'individu. En captant les activités individuelles et les flux de ressources dirigés vers la cuisine familiale, l'enquête propose des possibilités d'analyse de la sécurité alimentaire basées sur la déconstruction des unités classiques d'analyse (résidence, ménage, exploitation). Des communautés de production et de circulation sont alors établies pour pouvoir étudier les stratégies d'approvisionnement de la communauté de consommation, point de départ de l'étude de la sécurité alimentaire. Le questionnaire a été appliqué à 350 ménages dans une région d'étude au nord-ouest du Nicaragua. La collecte s'est faite par questionnaire papier et en entretien face-à-face entre avril et juin 2016. Des entretiens individuels complémentaires ont été conduits entre février et mai 2016. Les données ont été saisies entre juillet et octobre 2016, avant d'être contrôlées, nettoyées et préparées pour les analyses entre juin et octobre 2017 sur le logiciel SPSS. Les métadonnées ont été produites sur NESSTAR.
\end{abstract}

Mots clés : agriculture / migration / sécurité alimentaire / ménages / Nicaragua

\begin{abstract}
Exploring the links between agriculture, migration and food security: a survey of diversified, multi-situated farming households in Northwestern Nicaragua. This paper presents a survey conducted in northwestern Nicaragua, which aims at understanding how the flows of money and food generated by the diversified and multi-situated activities of farm households intervene in food access and perception of food security. In the study area, family agriculture develops in difficult agro-ecological conditions, and aims at producing staple food production and cattle ranching. In response to the lack of income, farm households have diversified their agricultural production with off-farm activities, locally and in migration. This diversification and multilocation allow them to generate income and food, essential for food security in regions at the origin. Social relationships between household members and beyond
\end{abstract}

\footnotetext{
*Auteur de correspondance : freguin@cirad.fr
} 
(extended family, neighborhood, and institutional actors) structure the flows of money and food. To capture these flows, the survey questionnaire, composed of three sections, is organized around a unique identification key: the individual. By capturing individual activities and resource flows directed towards the family kitchen, the survey offers possibilities for analyzing food security based on the deconstruction of conventional analysis units (residence, household, operation). Production and circulation communities are then established to study the supply strategies of the consumption community, which is the starting point for the analysis of food security. The questionnaire was applied to 350 households in a study area located in the North-west of Nicaragua. Data were gathered by paper questionnaire and face-to-face interview between April and June 2016. Additional individual interviews were conducted between February and May 2016. The data were entered between July and October 2016, before being checked, cleaned and prepared for analysis between June and October 2017 on the SPSS software. The metadata was produced on NESSTAR.

Keywords: agriculture / migration / food security / households / Nicaragua

\section{Introduction}

À partir d'une enquête de terrain menée au Nicaragua, cette contribution offre une réflexion théorique et propose une méthodologie pour analyser la sécurité alimentaire des ménages agricoles dans les sociétés du Sud. Quel que soit le contexte géographique, l'analyse de la sécurité alimentaire d'un ménage suppose de s'interroger à la fois sur ses manières d'accéder à l'alimentation et sur la nature de sa diète, que ce soit en termes de quantité, de diversité ou encore de qualité nutritionnelle. Selon la définition communément admise, la sécurité alimentaire est considérée comme assurée dès lors que « toutes les personnes, en tout temps, ont économiquement, socialement et physiquement accès à une alimentation suffisante, sûre et nutritive qui satisfait leurs besoins nutritionnels et leurs préférences alimentaires pour leur permettre de mener une vie active et saine» selon la définition proposée par la Conférence mondiale de l'alimentation de 1996.

Toutefois, la spécificité des ménages agricoles en milieu rural dans les sociétés au Sud (en comparaison par exemple avec celle de ménages citadins) est qu'ils sont eux-mêmes producteurs d'aliments qu'ils peuvent (ou non) consommer. De ce fait, la disponibilité alimentaire en lien avec le système de production développé sur l'exploitation agricole (elle-même déterminée par les facteurs de production de l'exploitation) conditionne largement les stratégies d'approvisionnement alimentaire du ménage (arbitrage entre autoconsommation, troc ou achat) et la nature de sa diète. En conséquence, les approches classiques de la sécurité alimentaire des ménages ruraux conçoivent leur schéma analytique à partir des interactions entre système productif agricole et sécurité alimentaire. D'un point de vue méthodologique, la plupart de ces approches considèrent que l'unité «ménage agricole» correspond à la superposition d'une famille nucléaire (ou élargie) et d'une exploitation agricole.

Les apports de ces travaux ont été et restent essentiels à la compréhension de la sécurité alimentaire des ménages agricoles. Nous considérons cependant qu'ils omettent certaines réalités relatives à ce que l'on pourrait appeler les ruralités "complexifiées ». Il convient en effet de relativiser l'exclusivité du secteur agricole dans nombre de sociétés rurales, en particulier celles où prédomine une agriculture familiale aux faibles dotations et à la faible productivité, qui sont marquées par la diversification des activités des membres des ménages (pluriactivité), et surtout par des migrations temporaires ou circulaires souvent anciennement installées. Beaucoup de travaux montrent l'importance de la diversification des activités des ménages (Ancey et Freguin-Gresh, 2014), ainsi que de la multi-localisation de leurs membres liée aux mobilités et aux migrations vers d'autres régions rurales ou urbaines, et vers d'autres pays (Cortès, 2000; Trousselle, 2012; Prunier, 2013; Freguin-Gresh et al., 2015). Par la diversification et la multilocalisation, les ménages ruraux ne vivent donc pas seulement de l'agriculture de l'exploitation familiale (la pluriactivité existe depuis longtemps à divers degrés dans les sociétés agraires). Autrement dit, nous sommes amenés à sortir d'une approche traditionnelle de la ruralité (c'est-à-dire agro-centrée) en interrogeant le caractère exclusivement agricole des ménages ruraux, et même le qualificatif «rural» des ménages, dans la mesure où les migrations, et la dispersion spatiale des familles qu'elles supposent, participent potentiellement des stratégies de subsistance des ménages, sous l'effet notamment de la circulation d'argent et d'aliments.

Il est paradoxal, à ce titre, que les triples liens entre agriculture, migration/circulations et sécurité alimentaire soient encore peu abordés dans la littérature, même si de nombreux travaux explorent les liens entre agriculture et alimentation en tenant compte des revenus extra-agricoles et des revenus reçus des membres des ménages en migration, incluant la dimension non marchande. Certes, des anthropologues, sociologues ou géographes ont décrypté les changements ou le maintien des habitudes alimentaires des groupes migrants, soit à destination, soit à leur retour (Crenn et Téchoueyres, 2010 ; Essomba et al., 2010 ; Ruf, 2010 ; AkcaySelcuk, 2017; Hunter-Adams, 2017) ou encore à partir de la circulation des aliments (Bouly de Lesdain, 2002; Adjamagbo et al., 2006; Eloy 2008). En revanche, le lien plus spécifique entre migrations et sécurité alimentaire est moins renseigné par la littérature, ou alors de façon plus récente (Crush, 2013; Knoll et al., 2017). Concernant les migrations internationales, le débat porte en particulier sur leurs impacts (négatifs ou positifs), sur les situations alimentaires des ménages/individus dans les pays de destination (Carney, 2017), ou dans les espaces de départ (Cortès, 2000; Crush, 2013). À ce titre, certains travaux en économie et géographie traitent de la question très spécifique des «food remittances» (remises alimentaires) dans les régions d'émigration (Lacroix, 2012; Kuuire et al., 2013; Atuoye et al., 2017).

Les résultats souvent divergents de ces travaux montrent combien la sécurité alimentaire des familles agricoles, 
notamment en contexte d'émigration, relève d'interactions et de processus complexes (Crush, 2013; Crush et Caesar, 2016; Crush et Caesar, 2017). Cette complexité analytique tient de toute évidence à la grande variabilité des contextes sociogéographiques et ruraux. Les résultats varient de plus selon les choix méthodologiques et les indicateurs retenus de la migration (incidence migratoire, transfert monétaire de la migration, etc.) et de la sécurité alimentaire (couverture calorique ou protéique, diversité de la consommation alimentaire, perception de l'insécurité, etc.).

\section{Proposition d'un cadre analytique}

L'enquête au cœur de ce data paper a été conçue et appliquée dans le cadre d'un projet de recherche à visée méthodologique portant sur la Sécurité ALImentaire au sein des Ménages Agricoles (projet SALIMA, métaprogramme GloFooDS Cirad-INRA). L'enquête SALIMA a été menée au nord-ouest du Nicaragua, dans une région rurale particulièrement marquée par les migrations internes et internationales. Elle vise à comprendre comment les flux d'argent et d'aliments générés par les activités économiques diversifiées et multilocalisées de familles agricoles interviennent dans l'accès aux aliments et dans la perception de la sécurité alimentaire.

Pour concevoir l'enquête, l'équipe s'est appuyé sur un cadre d'analyse pluridisciplinaire fondé sur la géographie, l'économie rurale et la sociologie, permettant d'étudier la complexité des stratégies socio-économiques et spatiales des familles, basées sur la diversification des activités sur et hors exploitation agricole de leurs membres, et incluant des activités en migration (de tout type de destination et durée). L'enjeu est de pouvoir rendre compte des flux multidirectionnels qui approvisionnent en aliments la cuisine familiale.

Ce cadre analytique suppose la déconstruction des unités d'analyse «classiques» dans l'approche de la sécurité alimentaire en milieu agricole, à savoir le ménage strictement associé à l'exploitation agricole. Dans cette recherche, nous définissons le ménage comme étant l'unité de résidence ou maison (casa en espagnol) où vit habituellement un groupe d'individus, liés généralement (mais pas nécessairement) par la parenté. Précisons qu'un ménage peut être composé d'une ou plusieurs familles nucléaires (définie comme le groupe formé par les parents et leurs enfants), qu'elle(s) soi(en)t au complet ou non (en cas de migration de l'un des membres par exemple, la famille nucléaire est incomplète). Dans le questionnaire, nous avons apporté une précision: habituellement correspond à une durée d'au moins 4 jours par semaine pendant plus de 6 mois par an, une durée fixée arbitrairement et qui correspond à celle généralement trouvée dans la littérature pour définir des migrants de longue durée. Parallèlement, nous définissons l'exploitation agricole comme une unité économique dédiée à la production agricole et à l'élevage, soumise à une direction unique, individuelle ou collective, comprenant l'équipement, les animaux et la terre (utilisée entièrement ou en partie), indépendamment de son mode de faire-valoir, de sa titularisation ou du statut juridique des parcelles (FAO, 2016). Dans la région d'étude, tous les ménages interrogés ont une exploitation agricole où ils réalisent des activités productives génératrices d'aliments et d'argent. Toutefois, des membres des ménages ont également d'autres activités productives hors exploitation, locales ou non.

Ces unités étant définies, nous concevons un cadre analytique qui repart de l'échelon individuel et qui positionne chaque individu dans des sphères d'appartenance. Comme illustré dans la figure 1, nous distinguons trois sphères:

- la sphère de consommation regroupe les individus qui mangent ensemble sous le même toit. Nous nous inspirons ici de la définition de la communauté de consommation de Gastellu (Gastellu, 1980) qui nous permet d'envisager la réalité des pratiques de consommation en œuvre dans la région agricole que nous étudions. En résidant ensemble, les membres d'un ménage partagent la plupart de leurs repas dans leur résidence. Mais il est possible que certains mangent hors de la maison et que d'autres individus, extérieurs au ménage, prennent des repas avec lui. Autrement dit, le contour de la sphère de consommation peut ne pas recouvrir les contours du ménage, ni ceux de la famille nucléaire ;

- la sphère de production regroupe les individus qui produisent des ressources (argent et aliments) permettant de nourrir les individus de la sphère de consommation. Soit les aliments proviennent de l'activité agricole sur l'exploitation familiale, soit ils sont achetés sur le marché grâce aux revenus tirés des activités productives (agricoles ou non, celles des membres du ménage ou des migrants). La sphère de production dépasse donc les contours de l'exploitation agricole, mais aussi ceux du ménage dans la mesure où elle capte les membres en migration de moyenne et longue durée (plus de 6 mois) quand ils envoient de l'argent à leur famille;

- la sphère de circulation regroupe les individus qui font circuler des aliments vers la sphère de consommation. Elle inclut les migrants qui envoient ou ramènent des aliments, ainsi que les individus extérieurs au ménage et à la sphère de production (dons ou troc avec les voisins, ou avec la famille élargie). Cette sphère tient compte aussi des acteurs des politiques publiques qui procèdent à des dons et à une distribution de biens qui participent à l'approvisionnement alimentaire des ménages (aliments, animaux d'élevage essentiellement).

Ces trois communautés sont donc les unités d'analyse au cœur du cadre proposé. La sphère de consommation est l'unité sociale de la mesure de la sécurité alimentaire, tandis que les deux autres sphères permettent de décrypter les stratégies d'approvisionnement alimentaire. Autrement dit, les trois sphères sont liées par des flux d'argent et d'aliments, associés aux activités productives des familles et aux dons et redistributions dont elles peuvent bénéficier, qui sont dirigés vers la cuisine familiale. Ces flux dépendent des rapports sociaux entre les membres des familles, et entre les familles et leur environnement social et institutionnel (voisinage, famille élargie, acteurs institutionnels). Pour caractériser ces rapports sociaux, nous nous inspirons de la contribution de Polanyi à la sociologie économique qui rend compte des dimensions de la marchandisation: administration domestique, rapports marchands monétaires ou non monétaires, rapports non marchands (Polanyi, 1983). Nous considérons que la sphère de production 


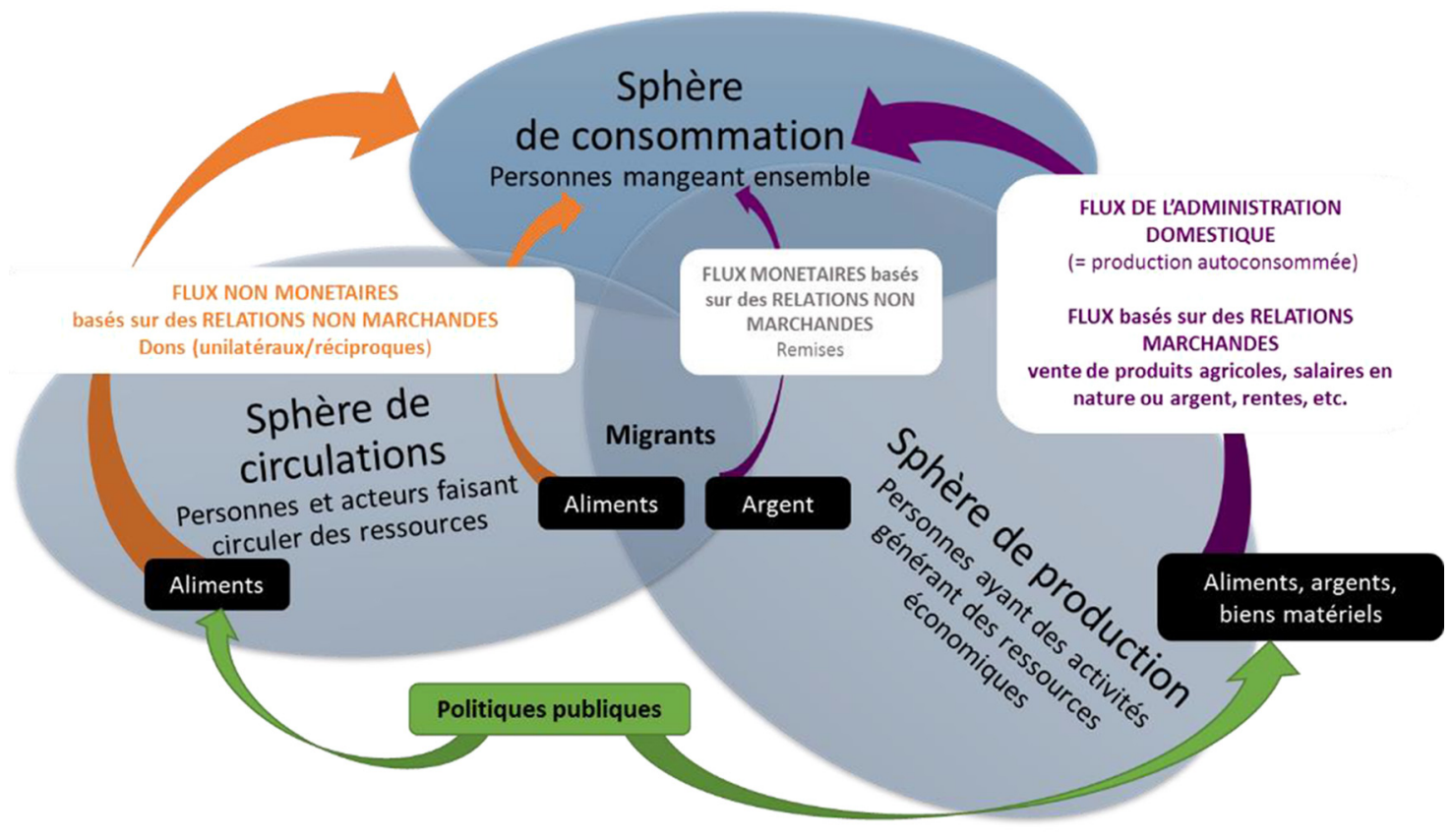

Fig. 1. Représentation schématique du cadre conceptuel et d'analyse du projet SALIMA au Nicaragua.

Fig. 1. Schematic figure of the conceptual and analytical framework of the SALIMA project in Nicaragua.

caractérise les flux correspondant soit à «l'administration domestique » de la famille (production de l'exploitation agricole autoconsommée), soit à des « rapports marchands », c'est-à-dire à des salaires et rentes en nature, à la vente de produits de l'exploitation agricole ou de produits naturels collectés, ou à des revenus liés au travail hors de l'exploitation familiale. Les transferts en argent des migrants renvoient eux à des « rapports non marchands monétaires ». La sphère de circulation, quant à elle, concerne les « rapports non monétaires et non marchands », à savoir les transferts alimentaires des migrants, mais aussi les dons (unilatéraux ou réciproques) de la famille élargie ou du voisinage, et la «redistribution» d'aliments (programmes publics et projets d'aide alimentaire).

En termes de collecte des données, l'enjeu du dispositif d'enquête a été de pouvoir cerner les contours des trois sphères. La démarche a consisté à partir de la sphère de consommation (donc de la cuisine familiale d'une résidence), pour identifier ensuite les individus qui contribuent aux flux de ressources produites (argent, biens, produits alimentaires) et aux flux de ressources qui circulent (dons, troc, aide alimentaire, etc.). L'application du cadre repose par ailleurs sur une démarche de recueil des données multi-niveaux (individu, ménage, exploitation agricole, maison) et qui combine plusieurs méthodes de collecte (focus groups, entretiens, questionnaires) permettant de caractériser le contexte, le fonctionnement des systèmes alimentaires familiaux et les rapports intra et supra-ménage sous-jacents au fonctionnement de ces systèmes (Fig. 2).

\section{Description des données}

\subsection{Choix de la région d'étude : caractéristiques générales et spécificités pour la recherche}

Le terrain d'étude se situe dans une région agricole du département de Chinandega, au nord-ouest du Nicaragua et à la frontière avec le Honduras. Nous avons choisi de conduire la recherche au Nicaragua en raison de l'importance de l'agriculture dans l'économie de ce pays et de la vulnérabilité de ce secteur, qui agissent sur le développement de stratégies familiales articulant l'agriculture avec d'autres activités, notamment en migration, et de l'incidence de cette situation sur la sécurité alimentaire.

Le Nicaragua est un pays à faible revenu d'Amérique centrale, dont l'histoire tumultueuse est liée aux crises sociales, politiques, économiques et aux catastrophes naturelles. Son économie repose largement sur l'agriculture, contrairement aux autres pays centroaméricains qui ont amorcé un changement structurel au $\mathrm{xx}^{\mathrm{e}}$ siècle : l'agriculture nicaraguayenne contribue à $15 \%$ du PIB selon les données de la Banque centrale; elle est le premier secteur économique avant l'exploitation minière $(14 \%)$ et le commerce $(11 \%)$. Dans un contexte de forte vulnérabilité liée à une ouverture précoce du pays aux échanges mondiaux, l'agriculture du Nicaragua a un faible niveau de développement technologique et est sensible aux variations climatiques (CEPAL, 2017). Cela se répercute sur le déséquilibre de la balance commerciale, sur la pauvreté et l'insécurité alimentaire (FAO et OPS, 2017). Conséquence de cette situation, le Nicaragua est un pays marqué par la forte incidence de la migration. Les migrations internes et internationales ont façonné la société et le territoire, avec des flux « entrants » de populations originaires de l'isthme centroaméricain et d'Amérique du Sud dès le peuplement du pays, des circulations internes (du Pacifique vers l'Atlantique, des campagnes vers les villes) et d'autres flux à la fois « circulants » et «sortants» vers de multiples destinations internationales (Baumeister, 2006; OIM, 2013). L'intensité de ces flux n'a fait que s'accroître au cours du $\mathrm{xx}^{\mathrm{e}}$ siècle.

Située dans le corridor sec centroaméricain, le terrain d'étude est soumis à un climat subhumide caractérisé par des températures moyennes comprises entre 20 et $25^{\circ} \mathrm{C}$, et une 


\begin{tabular}{|cl|}
\hline $\begin{array}{c}\text { Contexte biophysique, } \\
\text { économique, social et } \\
\text { institutionnel }\end{array}$ & $\begin{array}{l}\text { - Znonage biophysique et socio-éco } \\
\text { - Politiques publiques d'aide } \\
\text { alimentaire }\end{array}$ \\
\hline $\begin{array}{c}\text { Fonctionnement des } \\
\text { systèmes alimentaires } \\
\text { familiaux }\end{array}$ & $\begin{array}{l}\text { - Pratiques de consommation } \\
\text { - Pratiques d'approvisionnement } \\
\text { en aliments } \\
\rightarrow \text { Stratégies alimentaires }\end{array}$ \\
\hline $\begin{array}{c}\text { Rapports sociaux intra- } \\
\text { et supra-ménage }\end{array}$ & $\begin{array}{l}\text { - Administration domestique } \\
\text { - Rappón } \\
\text { (monétaire ou non) }\end{array}$ \\
\hline & \\
\hline
\end{tabular}

Fig. 2. Démarche multiniveaux permettant de collecter les informations nécessaires à l'application du cadre analytique. Fig. 2. Multilevel approach to gather the data needed to apply the analytical framework.

pluviométrie ne dépassant pas $2000 \mathrm{~mm}$ par an, avec des minimas autour de $700 \mathrm{~mm}$ et une saison sèche de novembre à avril. Des sécheresses et des inondations durcissent ces conditions lors d'évènements climatiques récurrents comme $\mathrm{El}$ Niño, qui a d'ailleurs sévi dans la région d'étude en 2015 et en 2016, année de référence de l'enquête. Ces conditions climatiques ont des variations locales en fonction de l'altitude: le sud-ouest du terrain d'étude (communes de Somotillo et Villa Nueva) est caractérisé par une topographie globalement plane mais faiblement ondulée (pentes $>15 \%$ ) et une faible altitude $(<200 \mathrm{~m})$. La température moyenne y est relativement élevée $\left(23-25^{\circ} \mathrm{C}\right)$ et la pluviométrie variable (de 700 à $1800 \mathrm{~mm}$ ). La saison sèche est marquée avec des risques de canicule sévère (températures pouvant dépasser $45^{\circ} \mathrm{C}$ ). Du fait de la topographie, les risques d'inondation en saison des pluies sont aussi élevés. Le nord-est du terrain d'étude (communes de San Pedro del Norte, Cinco Pinos, San Francisco del Norte et Santo Tomas del Norte), situé en altitude (200 à $700 \mathrm{~m}$ ), a une topographie plus accidentée (pentes jusqu'à $50 \%$ ) et est soumis à un climat tropical subhumide d'altitude (pluviométrie pouvant atteindre $2000 \mathrm{~mm}$, températures moyennes plus fraîches, $20-23^{\circ} \mathrm{C}$ ). Ces variations locales entraînent des conditions agro-écologiques différenciées en plaine et en montagne, et influencent l'exploitation agricole (choix des cultures et des cycles de production, possibilité de développement de l'élevage bovin extensif).

À cause des limitations climatiques et agroécologiques, la région a été relativement épargnée par la concentration foncière et le développement d'exploitations capitalistes à grande échelle. Ces exploitations, qui se sont précocement insérées dans les échanges internationaux en exportant des cultures industrielles (coton, canne à sucre, bananes) ou les produits de l'élevage bovin extensif, se sont davantage situées dans le sud du département où la topographie est plus favorable à l'installation de plantations mécanisables, où les sols volcaniques sont plus fertiles et où les aménagements (drainage) ont limité les risques d'inondation. Souvent expulsées de ces zones à fort potentiel agricole faisant l'objet de l'appui des politiques publiques, des familles sans terre ou d'agriculteurs aux ressources limitées se sont installées dans la région d'étude, entraînant le développement de l'agriculture familiale. Ces familles ont alors cultivé des vivres (maïs, sorgho, haricots), d'autres cultures de diversification destinées à la vente (sésame, pastèque, fruits) et pratiqué l'élevage bovin extensif sur des pâturages peu productifs. Toutefois, la production agricole est affectée par les conditions agroécologiques défavorables décrites précédemment, ce qui se traduit par une vulnérabilité alimentaire et la recherche de revenus complémentaires localement ou en migration. Pour cela, la région d'étude présente un intérêt particulier pour étudier l'incidence des migrations sur l'agriculture et la sécurité alimentaire.

\subsection{Méthode d'échantillonnage des ménages étudiés}

L'échantillon des ménages étudiés n'est pas statistiquement représentatif, étant donné qu'il n'existe pas de base de sondage suffisamment récente comme pourrait l'être un recensement de la population. Le dernier recensement de population datant de 2005 , nous avons considéré, vu les taux de croissance démographique au niveau national et la forte incidence de la migration dans la région d'étude, qu'il n'aurait pas été pertinent d'utiliser ce recensement de population comme base de sondage, ni ses projections les plus récentes ; en effet, selon les experts de l'Institut nicaraguayen d'information de développement (INIDE) - organisme producteur de ces projections -, elles sont éloignées de la réalité, qui reste largement méconnue.

Pour réaliser l'échantillonnage, nous avons donc procédé en deux étapes. Nous avons d'abord sélectionné de manière raisonnée des sites d'enquête (localités ou villages), puis nous avons tiré de manière aléatoire les ménages à interroger sur chaque site, sur la base d'une taille d'échantillon proportionnelle à la taille démographique des villages. Pour choisir les sites, nous nous sommes basés sur des critères construits sur la base d'informations existantes et sur d'autres établis à dires d'experts. Les critères sont les suivants: 1) conditions climatiques et agroécologiques synthétisées dans un zonage 
S. Fréguin-Gresh et al. : Cah. Agric. 2019, 28, 9

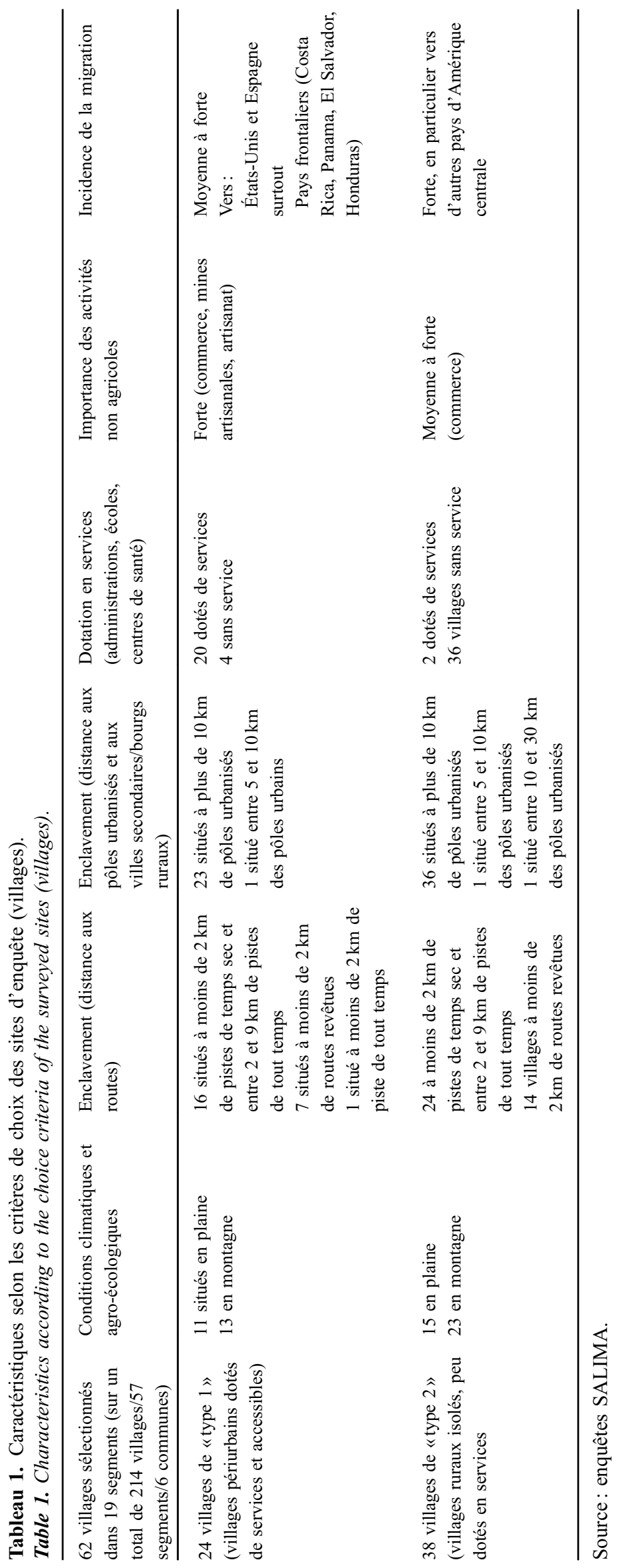

Page 6 de 12 
Distribution des ménages enquêtés dans les
villages de "Type 1"

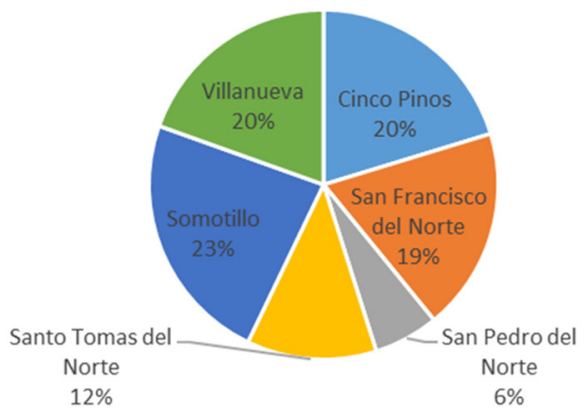

Distribution des ménages enquêtés dans les villages de "Type 2 "

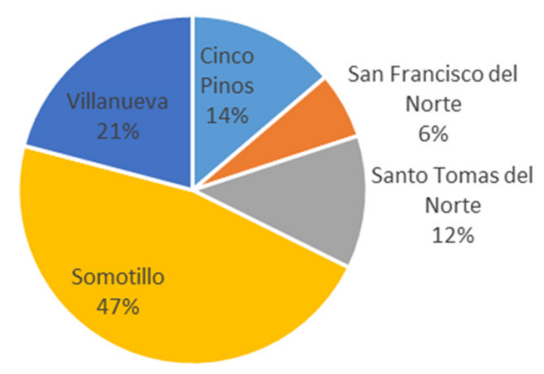

Fig. 3. Distribution de l'échantillon par commune selon les deux types de villages retenus.

Fig. 3. Distribution of the sample by municipality according to the two types of selected villages.

(plaine/montagne), enclavement traduit par la distance aux routes, aux pôles urbanisés et aux infrastructures de base (services administratifs, de santé et écoles) - trois critères pour lesquels nous avions des données secondaires spatialisables; et 2) importance des activités non agricoles et incidence de la migration, indicateurs fournis par des personnes ressources de la région d'étude.

Etant donné que l'échelle de la commune ne nous permettait pas de définir de manière précise des sites d'enquêtes (villages, appelés en espagnol communautés) différenciés sur la base de ces critères, nous avons opté pour une maille correspondant aux segments du dernier recensement agricole de 2011. En utilisant cette maille, qui regroupe un nombre variable de villages aux caractéristiques proches (selon nos critères), nous avons pu déterminer grâce au recensement agricole un nombre de ménages à interroger par segment de recensement $(10 \%$ du nombre de ménages agricoles recensés dans un segment de recensement en 2011). Notons que le nombre de village inclus dans un segment de recensement dépend de la concentration et de la distribution de la population dans cet espace. Nous avons appliqué cette taille d'échantillon dans une sélection aléatoire de villages au sein de chaque segment. L'enquête ayant montré que les ménages interrogés, choisis aléatoirement dans chaque village, avaient tous une exploitation agricole, il s'avère que ce choix était pertinent pour avoir une bonne approximation de la réalité (Tab. 1, Fig. 3 et 4).

\subsection{Unités d'observation, unités d'analyse et indicateurs de sécurité alimentaire utilisés}

L'enjeu méthodologique du projet SALIMA est de discuter la pertinence des unités d'observation et d'analyse utilisées pour étudier la sécurité alimentaire. Le travail conduit au Nicaragua propose de construire des unités d'analyse pertinentes au regard des situations empiriques observées. Cela a des conséquences sur la manière de conduire la collecte de données: enquête adressée à qui ? Renseignant sur qui (individu, ménage, collectif familial, etc.) ? Concernant quels types de données (quoi ?).

Dans cette recherche, nous appréhendons la sécurité alimentaire surtout dans sa dimension «accès» et, dans une moindre mesure, dans sa dimension «disponibilité ». Nous utilisons plusieurs indicateurs (Leroy et al., 2015):

- l'échelle d'accès à l'insécurité alimentaire du ménage (Household Food Insecurity Access Scale, HFIAS), qui est un score de 0 à 27 visant à estimer la prévalence de l'insécurité alimentaire ( 0 étant la situation de sécurité alimentaire) (Deitchler et al., 2010). Ce score est fondé sur des réponses en fréquence à 9 questions qui renvoient aux domaines universels et aux sous-domaines de l'expérience d'insuffisance d'aliments (au cours des 4 semaines avant l'enquête) et au domaine plus spécifique du manque d'accès aux aliments;

- le score de diversité de la diète à l'échelle du ménage (Household Dietary Diversity Score, HDDS), qui est aussi un indicateur d'accès aux aliments, qui renvoie plus spécifiquement à la qualité et la quantité de cet accès à l'échelle du ménage, l'accès étant défini comme la capacité de celui-ci à acquérir des aliments en qualité et quantité suffisante pour satisfaire les besoins en nutriments des membres du ménage pour qu'ils mènent une vie productive (Kennedy, 2009). Ce score de 0 à 12 (12 étant le maximum de diversité possible) est fondé sur le calcul du nombre de groupes alimentaires consommés (sur une base de 12 groupes) sur une période donnée (en théorie $24 \mathrm{~h}$, mais dans notre enquête, la semaine précédant l'enquête);

- la quantité et la diversité d'aliments - plus largement de produits agricoles - produits sur l'exploitation agricole, et obtenus par des circulations (transfert d'aliments, dons), mais aussi l'argent gagné par la sphère productive pouvant être utilisé pour l'alimentation.

Le questionnaire est structuré en trois sections thématiques: démographie et habitat, activités productives hors exploitation et sécurité alimentaire, activités agricoles (Fig. 5). Les identifiants permettant de relier ces trois sections sont le numéro (unique) attribué au ménage et le numéro (unique) attribué aux individus recensés. Pour pouvoir identifier les individus appartenant aux trois sphères de notre cadre d'analyse (consommation, production, circulation), nous avons collecté des données à plusieurs niveaux d'observation : i) le ménage (ensemble des individus vivant habituellement sous le même toit); ii) l'individu (membre du ménage ou hors 


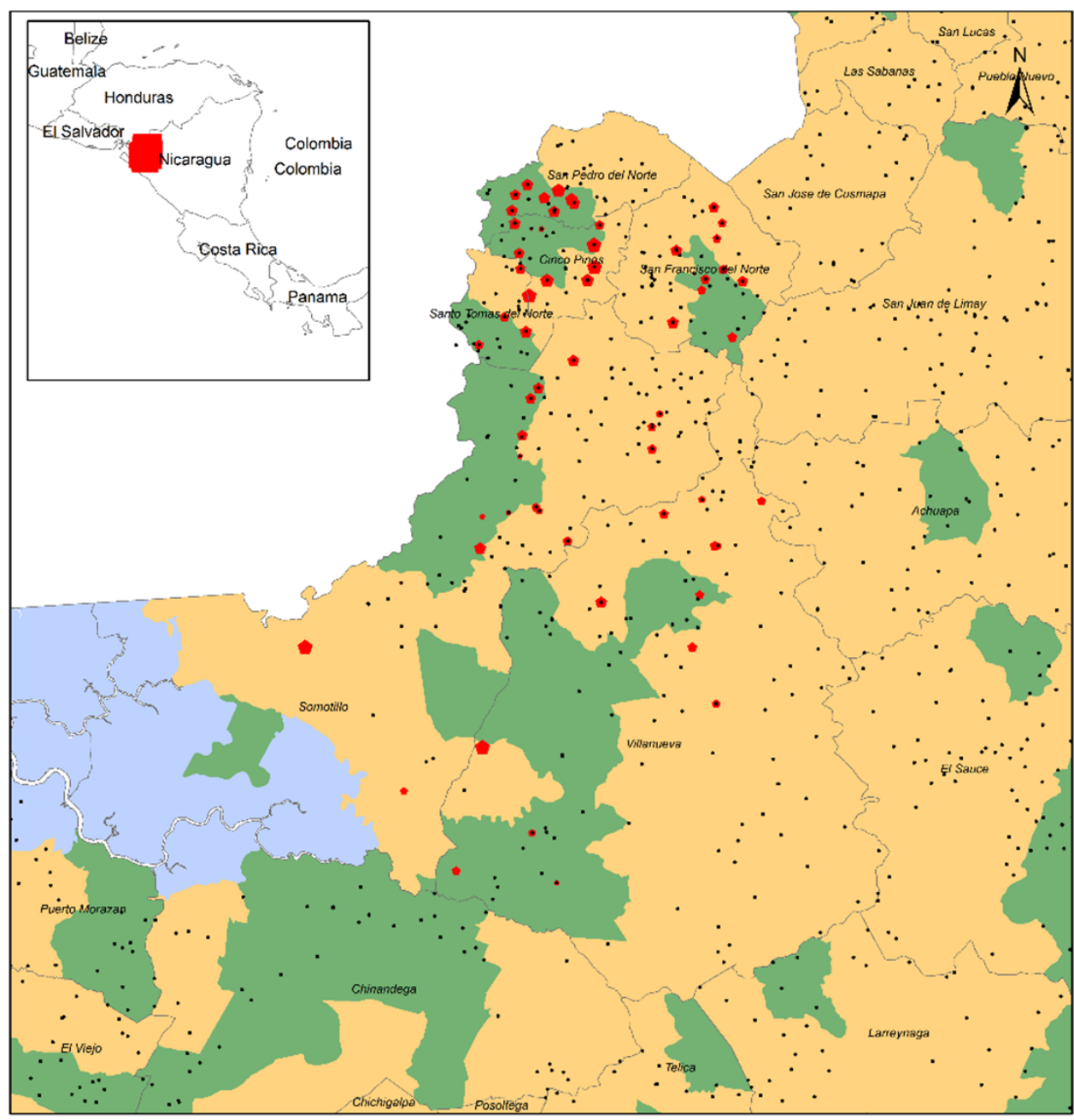

\section{Légende}

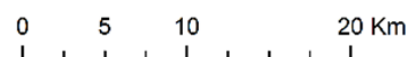

- Villages (communautés rurales)

\Limites administratives des communes

\section{Nombre de ménages enquêtés}

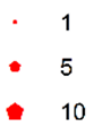

\section{Villages de "Type 1" \\ Villages de "Type 2" \\ Zone de l'Estuaire Real non peuplée}

Fig. 4. Carte de localisation et de distribution des ménages étudiés selon les deux types de villages retenus.

Fig. 4. Location and distribution map of the surveyed households according to the two types of selected villages.

ménage, membre avec lien de parenté ou non); iii) l'exploitation agricole et la parcelle. La figure 5 montre comment s'organisent dans le questionnaire les données à chaque niveau d'observation.

La combinaison de ces différentes unités d'observation (individus, ménage et exploitation) a permis non seulement de capter les flux d'argent et d'aliments entrants/sortants mais également de voir les effets de superposition (ou non) entre ménage-famille-exploitation agricole. Or, après analyse des données, il s'est avéré que i) l'unité ménage coïncidait avec une seule unité familiale (famille nucléaire et membres étant en migration au moment des enquêtes); ii) l'unité «ménage» 


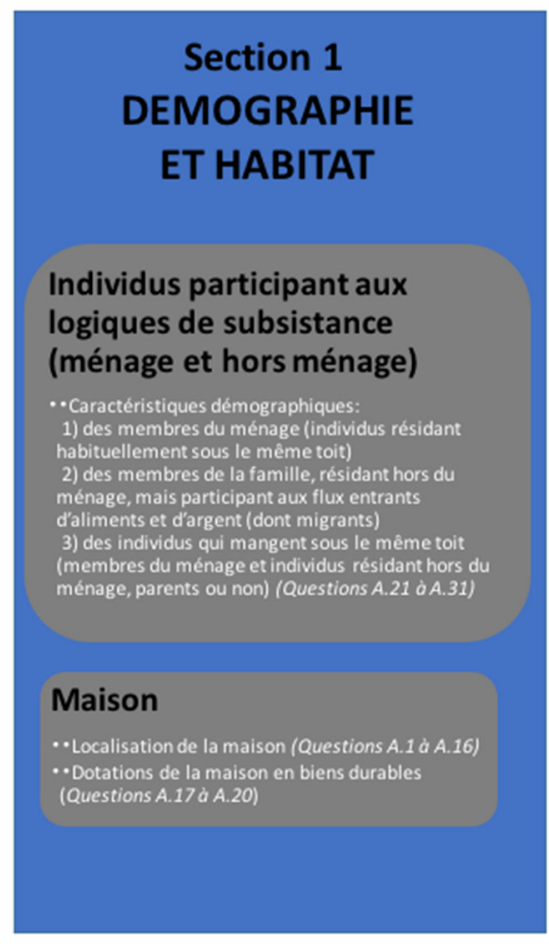

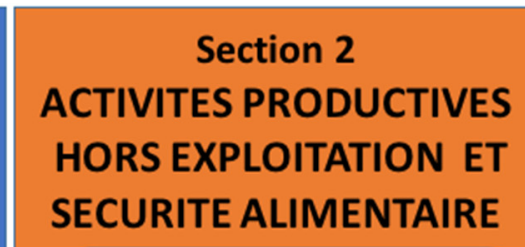

Individus participant aux

logiques de subsistance

(ménage et hors ménage)

- "Les activités productives hors exploitation

agricole (Questions B.1ò B8) et en migration

(Questions B.9à B.16)

Ménage

-.Les relations du ménage avec son
environnement (Questions 8.17 a B.33)

environnement (Questions B.17 a B.33)
- -Les dépenses (Questions B.34 à B.39)

- - - Le stockage des vivres (Questions B.41 à B.46)

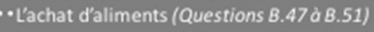

- - La consommation d'aliments (Questions B.52

- La consommats
$\dot{a} B .59) \rightarrow$ HDOS

- "-Léchelle de perception de linsécurité

alimentaire (Questions $B .62$ à B.70) $\rightarrow$ HFIAS

- - Les stratégies d'adaptation à l'insécurité

alimentaire (Question B.71)

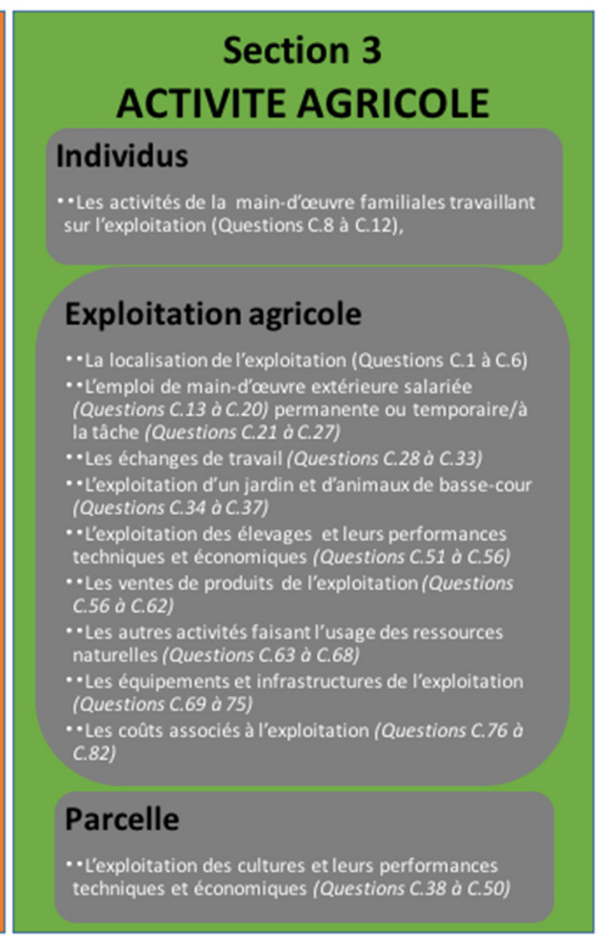

Fig. 5. Organisation des données dans le questionnaire.

Fig. 5. Organization of the data in the questionnaire.

coïncidait avec l'unité exploitation agricole (sachant que dans cette région le ménage n'a qu'une seule exploitation). De ce fait, nous avons pu organiser les données ménage et exploitation dans une base de données unique (identifiant = ménage). Les données recueillies à l'échelle de la parcelle ont également pu être synthétisées avec des indicateurs techniques et économiques dans cette base ménage. En revanche, nous avons conservé les informations recueillies à un niveau individuel dans une base séparée, même si des données ont pu permettre la création de variables synthétiques insérées dans la base ménage. De cette manière, nous avons pu identifier les individus selon leur appartenance aux différentes sphères d'analyse (consommation, de production, de circulation), certains individus pouvant appartenir à une, deux ou trois sphères.

Au sein de chaque ménage, nous avons identifié le chef de ménage qui a été interrogé pour répondre aux questions des sections 1 et 2 , sauf pour les questions relatives à l'achat et à la consommation alimentaire, pour lesquelles la personne responsable de ces tâches a été interrogée (généralement l'épouse du chef de ménage quand celui-ci était un homme). Pour la section 3, relative à l'exploitation agricole, c'est le responsable de la direction de l'exploitation agricole (le plus souvent le chef de ménage, mais parfois son fils) qui a été interrogé.

\subsection{Couverture temporelle de l'enquête}

Nous avons collecté les données entre fin février et mi-juin 2016: des entretiens ont été menés spécifiquement entre février et avril ; l'administration du questionnaire s'est faite sur la période d'avril à juin, en parallèle à la réalisation de 7 focus groups.

L'enquête par questionnaire porte sur le cycle de production agricole de l'année précédant l'enquête (cycle allant d'avril 2015 à avril 2016). Sur cette période de référence, ont été recensés : les activités, la main-d'œuvre agricole, les migrations, les résultats économiques des activités et en particulier dans la production agricole, les dépenses exceptionnelles et celles de l'exploitation, la main-d'œuvre agricole extérieure (temporaire ou permanente) et les relations de travail non rémunéré. Les données structurelles de la maison, du ménage et de l'exploitation renvoient à l'organisation socio-spatiale du ménage (localisation et distances de la maison et de l'exploitation agricole aux lieux d'achats, à la route selon les moyens de transport utilisés), aux caractéristiques de la maison, de la cuisine et de l'exploitation (dotation en biens durables), aux caractéristiques des individus (âge, genre, éducation). Ces données concernent la situation au moment de l'enquête.

D'autres informations du questionnaire concernent d'autres unités temporelles. Ainsi, les données sur la consommation telles que les achats d'aliments (quantité, coût), les aliments consommés par la communauté de consommation (nature et origine) et les dépenses alimentaires concernent la semaine précédant l'enquête. Les 9 questions sur la prévalence de l'insécurité alimentaire de l'échelle de la composante d'accès de l'insécurité alimentaire dans le ménage (Coates et al., 2007) concernent les 4 semaines avant l'enquête. 


\subsection{Population couverte par l'enquête}

La population de l'enquête est constituée de l'ensemble des individus qui : 1) participent aux repas du ménage (sphère de consommation) ; 2) participent aux activités productives sur l'exploitation familiale et; 3 ) hors exploitation, que ce soit localement ou en migration (sphère de production et de circulation). Dans l'enquête, les individus apparentés au ménage et non productifs (cas des enfants ou des individus n'ayant pas d'activité génératrice de ressources), qu'ils résident sous le même toit ou qu'ils soient en migration, sont aussi concernés par l'enquête, même s'ils n'appartiennent à aucune des sphères analysées. Au total, le questionnaire a été appliqué dans 350 résidences (343 questionnaires ont été conservés pour l'analyse après apurement), ce qui concerne un total de 1990 individus.

\section{Méthodologie}

\subsection{Concepteurs de l'enquête et autres contributeurs}

L'UMR ART-Dev (Acteurs ressources et territoires pour le développement), en particulier des chercheuses du Centre de coopération internationale en recherche agronomique pour le développement (CIRAD) et de l'Université Paul Valéry à Montpellier, est à l'origine de la conception de l'enquête et des instruments de collecte des données. Des chercheuses du CIRAD de l'UMR MOISA (Marchés, organisations, institutions et stratégies d'acteurs), impliquées dans le projet SALIMA sur un autre terrain, ont joué un rôle important dans la conception du questionnaire de l'enquête. Il est utile de mentionner que l'élaboration du questionnaire s'est appuyée sur un questionnaire existant (Projet MEDINA), qui a été retravaillé et adapté aux problématiques du projet SALIMA au Nicaragua. De même, des liens existent avec le questionnaire SALIMA Madagascar, qui a été conçu après l'enquête au Nicaragua.

\subsection{Collecteur des données}

Des assistantes de recherche de l'Institut de recherche et de développement local Nitlapan de l'Université centroaméricaine de Managua, Nicaragua, ont été responsables de la supervision de l'enquête par questionnaire et des focus groups. Une stagiaire de Master de l'Université Paul Valery (BanoviezUrrutia, 2016), a conduit des entretiens qualitatifs complémentaires auprès de 35 familles dans deux villages de la région d'étude, qui ont permis des ajustements importants de la méthodologie. L'application des questionnaires auprès des ménages s'est faite en espagnol, par des enquêteurs (femmes et hommes, en proportion égale) recrutés dans la région d'étude et formés pour l'enquête.

\subsection{Producteur des données}

Le producteur des données est le projet SALIMA Nicaragua, financé par le métaprogramme INRA-CIRAD GLOFOODS (AMI, 2015).

\subsection{Mode de collecte et administration des questionnaires}

\subsubsection{Phase pilote}

La phase pilote de l'enquête s'est faite en deux étapes. Tout d'abord, une étudiante de Master a réalisé un travail de terrain entre février et mai 2016 dans la région d'étude. Ce travail de recherche, dont l'objectif était de comprendre les pratiques de consommation et d'approvisionnement de ménages agricoles ayant des migrants dans la région d'étude, a permis la collecte de données auprès de 35 ménages sélectionnés sur le critère d'avoir des migrants dans deux villages de la région d'étude (les villages ont été inclus dans l'échantillonnage, mais pas les ménages). Ce stage intègre la phase pilote puisqu'il a été réalisé en parallèle de la conception des questionnaires et que ces ajustements ont été réalisés en fonction des observations et informations collectées sur le terrain. Une fois le questionnaire conçu, en espagnol, un premier test a été conduit par les superviseuses de l'Institut Nitlapan en mars 2016 auprès de ménages résidant à proximité de la capitale Managua, test à la suite duquel des ajustements au questionnaire ont aussi été réalisés.

\subsubsection{Formation des superviseuses d'enquêtes et des enquêteurs}

La formation des deux superviseuses, assistantes de recherche de l'Institut Nitlapan, s'est faite entre janvier et février 2016, en parallèle de la conception des questionnaires et de la précision des concepts utilisés. Les deux superviseuses ont ensuite formé six enquêteurs et enquêtrices employés spécifiquement pour la collecte qui s'est faite en espagnol, qui sont tous et toutes originaires des communes étudiées et interviennent dans le cadre de projets de développement local. Les superviseuses ont aussi été formées à la saisie des données sur le logiciel ACCESS en parallèle à la collecte sur le terrain.

\subsubsection{Type d'instrument et procédure de collecte}

La conduite d'un travail de terrain qualitatif en amont de l'application du questionnaire avec le stage de Master 1 nous a permis d'élaborer un questionnaire directif structuré (fermé). Toutefois, nous avons insisté, lors de la formation à l'outil, sur la nécessité de collecter les réponses non anticipées que nous avons ensuite codées lors de la saisie, compte tenu du caractère exploratoire de la recherche. Avant la visite des villages, les superviseuses du projet ont systématiquement présenté les objectifs du projet de recherche aux autorités élues (mairies) et aux leaders des villages afin de faciliter le travail des enquêteurs et de s'assurer de l'accord pour la conduite du travail de terrain. Notons que la sélection des ménages s'est faite par un tirage aléatoire des maisons dans chaque village sélectionné.

\subsection{Caractéristiques de la collecte sur le terrain}

Le temps d'administration des trois sections du questionnaire a varié de 1 à 3 heures, en fonction du nombre d'individus concernés, de la diversité de leurs activités sur et hors exploitation et de la diversité des aliments consommés. Étant donné que la période d'enquête a aussi correspondu au début 
de la saison des pluies et que certains des sites d'enquête sont rapidement inaccessibles à cette saison, nous avons convenu de débuter la collecte dans la zone de montagne et dans les villages les plus inaccessibles (certaines maisons n'étant accessibles qu'à pied par ailleurs), et de terminer la collecte dans les villages de la plaine les plus proches de la route panaméricaine. Pour se rendre dans certains villages, des motos ont été louées car les pistes n'étaient pas praticables en voiture et parfois, les enquêteurs ont dû accéder aux résidences à pied.

\subsection{Saisie des questionnaires}

La saisie des questionnaires s'est faite sur la base d'un masque de saisie élaboré sur ACCESS entre mai et juillet. La conception de la base ACCESS, organisée autour de 96 tables liées par clefs primaires (identifiant unique du ménage et dans certains cas identifiants unique de l'individu ou de la parcelle), a nécessité plusieurs ajustements en raison de la saisie de réponses aux questionnaires qui n'étaient pas pré-codées.

\subsection{Nettoyage des données}

Le nettoyage des données s'est opéré sur SPSS après que les données ont été exportées. Ce nettoyage s'est fait en deux phases. Une première phase de nettoyage a été effectuée d'août à octobre 2016 par les superviseuses de l'enquête pour vérifier certaines incohérences et la correspondance avec les questionnaires papiers; puis une deuxième phase de nettoyage s'est faite de juin à octobre 2017. L'essentiel des analyses a été conduit sous SPSS 20 et sous SPAD; certaines analyses ont aussi été menées sous STATA 12. C'est à l'issue de ces deux phases d'apurement que 7 ménages ont été supprimés de la base finale qui ne compte que 343 ménages agricoles.

\section{Description des fichiers de données et localisation des métadonnées}

Freguin-Gresh Sandrine, 2019. Données d'enquêtes Agriculture, Sécurité Alimentaire et Migration SALIMA Nicaragua 2016. DOI: 10.18167/DVN1/CI43N3. CIRAD Dataverse, DRAFT VERSION.

\section{Potentiel de réutilisation des données et transfert de méthode}

Ce projet pilote s'est donné pour objectif d'appréhender la sécurité alimentaire des ménages agricoles depuis les pratiques d'approvisionnement en aliments des cuisines de ces ménages. Le cadre analytique et la méthodologie développée permettent de rendre compte des stratégies complexes fondées sur des flux multidirectionnels, dont ceux liés à la migration et aux politiques publiques, qui jouent un rôle considérable pour expliquer l'insécurité alimentaire.

Bien que l'échantillon ne soit pas statistiquement représentatif, il est possible d'extrapoler les résultats à partir de la structure et du fonctionnement (combinaison de pratiques) des exploitations agricoles des ménages étudiés grâce à l'utilisation du IV CENAGRO (recensement général de l'agriculture du Nicaragua datant de 2011). Notons que ce recensement permet d'analyser la structure démographique des familles associées aux exploitations agricoles, une autre dimension qui permettrait d'extrapoler les résultats. Toutefois, étant donné qu'il date de 2011 et qu'il n'était pas possible de localiser de manière précise (à l'échelle des villages) les exploitations agricoles du recensement, nous n'avons pas voulu redresser l'échantillon bien qu'il ait été possible le faire. De plus, il existe au Nicaragua d'autres enquêtes récurrentes nationales sur les moyens d'existence. Parmi ces enquêtes récurrentes, celle de 2014 par exemple, la plus proche de l'enquête SALIMA, fournit une caractérisation des systèmes d'activités et de revenus des ménages en incluant les questions migratoires et les structures des ménages; elle donne donc aussi une possibilité d'extrapolation, sous réserve que l'on connaisse la localisation précise (villages) des ménages étudiés (information qui n'est pas en accès public au Nicaragua). Pour des raisons identiques à celles du recensement, nous n'avons pas souhaité redresser l'échantillon.

Au-delà de la réutilisation des données, nous pensons que l'intérêt de ce projet réside dans le cadre analytique et la méthodologie en tant que telle et, de ce fait, dans la manière de concevoir la collecte et l'analyse des données. Sous réserve de son adaptation à chaque cas d'étude, le dispositif $\mathrm{du}$ projet SALIMA Nicaragua a un fort potentiel de réutilisation dans l'étude de l'insécurité alimentaire dans des sociétés agricoles et rurales où la migration est forte et structure le fonctionnement et les stratégies socio-économiques des ménages.

\section{Références}

Adjamagbo A, Delaunay V, Lévi P, Ndiaye O. 2006. Comment les ménages d'une zone rurale du Sénégal gèrent-ils leurs ressources? Études rurales 177: 69-90.

Akcay-Selcuk KA. 2017. Remittances and calorie consumption nexus in Algeria. International Migration 55(4): 103-117.

Ancey V, Freguin-Gresh S. 2014. Les familles, le travail et l'exploitation agricole. In: Sourisseau JM, ed. Agricultures familiales et mondes à venir. Versailles, France: Quae, pp. 61-74.

Atuoye NK, Kuuire ZV, Kangmennaang J, Antabe R, Luginaah I. 2017. Residential remittances and food security in the Upper West Region of Ghana. International Migration 55(4): 18-34.

Banoviez-Urrutia V. 2016. El sistema alimentario familiar en dos comunidades del Norte de Chinandega (Nicaragua). À partir de las prácticas de consumo y abastecimiento en los hogares agropecuarios, ¿qué estrategias frente a las vulnerabilidades alimentarias? Master 1, Université Paul Valéry, Montpellier 3.

Baumeister E. 2006. Migración internacional y desarrollo en Nicaragua. Acuerdo de Cooperación CELADE-FNUAP (Fondo de Población de las Naciones Unidas, Oficina de Nicaragua) Centro Latinoamericano y Caribeño de Demografía (CELADE) - División de Población.

Bouly de Lesdain S. 2002. Alimentation et migration, une définition spatiale. In: Garabuau-Moussaoui I, Palomares E, Desjeux D, eds. Alimentations contemporaines. Paris : L'Harmattan, pp. 173-189.

Carney AM. 2017. Back there we had nothing to eat: The case of transnational food insecurity. International Migration 55(4): 64-77.

CEPAL. 2017. Seguridad alimentaria y nutricional en Centroamérica y la República Dominicana: explorando los retos con una 
perspectiva sistémica. Ciudad de México, Comisión Económica para América Latina y el Caribe: 190.

Coates J, Swindale A, Bilinsky P. 2007. Household Food Insecurity Access Scale (HFIAS) for measurement of household food access: Indicator guide. Washington DC, Food and Nutrition Technical Assistance Project, Academy for Educational Development.

Cortès G. 2000. Partir pour rester. Survie et mutations des sociétés paysannes andines. Paris: IRD, $413 \mathrm{p}$.

Crenn C, Téchoueyres I. 2010. Les pratiques alimentaires des «élites » malgaches installées en France. Hommes et migrations 1283: $150-161$.

Crush J. 2013. Linking food security, migration and development. International Migration 51(5): 61-75.

Crush J, Caesar M. 2016. Food remittances: Migration and food security in Africa. Migration Policy Series 72: 51.

Crush J, Caesar M. 2017. Introduction: Cultivating the migrationfood security nexus. International Migration 55(4): 10-17.

Deitchler M, Ballard T, Swindale A, Coates J. 2010. Validation of a measure of household hunger for cross-cultural use. Washington, DC, TUFTS University. USAID. Food and Nutrition Technical Assistance II Project (FANTA-2). FHI 360: 76.

Eloy L. 2008. Diversité alimentaire et urbanisation. Le rôle des mobilités circulaires des Amérindiens dans le Nord-Ouest Amazonien. Anthropology of Food S4: 17.

Essomba JM, Edjenguèlè M, Pasquet $\mathrm{P}$, Hubert A. 2010. Migrations et pratiques culinaires. Hommes et migrations 1283: 136-149.

FAO. 2016. Programme mondial de recensement de l'agriculture 2020. Collection FAO : département Statistiques. Rome, Food and Agriculture Organization (FAO). Volume 1: Programme, concepts et définitions, $34 \mathrm{p}$.

FAO et OPS. 2017. Panorama de la Seguridad Alimentaria y Nutricional en América Latina y el Caribe. Santiago de Chile, Naciones Unidas.

Freguin-Gresh S, Cortes G, Sourisseau JM, Guetat-Bernard H. 2015. Le système familial multilocalisé. Proposition analytique et méthodologique pour interroger les liens entre migrations et développement rural au Sud. Mondes en développement 43(172): $13-32$.
Gastellu JM. 1980. Mais où sont ces unités économiques que nos amis cherchent tant en Afrique? Cahiers ORSTOM. Série sciences humaines 17(1-2): 3-11.

Hunter-Adams J. 2017. Exploring Perceptions of the Food Environment Amongst Congolese, Somalis and Zimbabweans Living in Cape Town. International Migration 55(4): 78-87.

Kennedy GL. 2009. Evaluation of dietary diversity scores for assessment of micronutrient intake and food security in Developing countries. $\mathrm{PhD}$, Wageningen University.

Knoll A, Rampa F, Torres C, Bizzotto Molina P, Cascone N. 2017. The nexus between food and nutrition security, and migration: Clarifying the debate and charting a way forward. Discussion paper 212. Maastricht: Ecdpm.

Kuuire V, Mkandawire P, Arku G, Luginaah I. 2013. Abandoning'farms in search of food: Food remittance and household food security in Ghana. African Geographical Review 32(2): 125-139.

Lacroix T. 2012. Migration, rural development, poverty and food security: A comparative perspective. University of Oxford, FAO/ International Migration Institute, $53 \mathrm{p}$.

Leroy J, Ruel L, Marie E, Frongillo A, Harris J, Ballard TJ. 2015. Measuring the food access dimension of food security: A critical review and mapping of indicators. Food and Nutrition Bulletin 36 (2): 167-195.

OIM. 2013. Perfil migratorio de Nicaragua 2012. Managua, Nicaragua, Organización Internacional para las Migraciones (OIM), 218 p.

Polanyi K. 1983. La grande transformation. Aux origines politiques et économiques de notre temps (édition originale 1944). Paris, France : Gallimard.

Prunier D. 2013. De nouvelles ruralités en Amérique centrale? Dynamiques de mobilité, ressources et organisations familiales. PhD, Université Paris Diderot-Paris VII.

Ruf F. 2010. Les Baoulé ne sont pas des oiseaux pour manger du riz. Alimentation, migrations et écologie du sud ouest ivoirien. Anthropology of Food 7: 16.

Trousselle A. 2012. Les mobilités rurales au Nicaragua, au prisme du transnationalisme et des modèles d'archipel familiaux: caractéristiques et perspectives. Master 2, ISTOM, Angers.

Citation de l'article : Fréguin-Gresh S, Padilla L, Pavon K, Elizondo D, Cortès G, Banoviez Urrutia V, Lourme Ruiz A, Dury S, Bouquet E. 2019. Explorer les liens entre agriculture, migration et sécurité alimentaire: une enquête auprès de ménages agricoles diversifiés et multilocalisés du nord-ouest du Nicaragua. Cah. Agric. 28: 9. 